\title{
Effect of beam density on nonlinear amplification of inverse-bremsstrahlung electron acceleration
}

\author{
A. C. J. PAES, ${ }^{1}$ M. VIRGÍNIA A LVES, ${ }^{2}$ R. M. O. GALVÃ $O,{ }^{3}$ \\ A. SERBET O ${ }^{4}$ and M.S. HUSSEIN ${ }^{3}$ \\ ${ }^{1}$ IEAv, Centro Técnico Aeroespacial, 12231-590 São José dos Campos, SP, Brasil \\ ${ }^{2}$ Instituto Nacional de Pesquisas Espaciais, PO Box 515, 12201-970 \\ São José dos Campos, SP, Brasil \\ ${ }^{3}$ Instituto de Física, Universidade de São Paulo, 05359-970 São Paulo, Brasil \\ ${ }^{4}$ Instituto de Física, Universidade Federal Fluminense, Av. Litorânea s/n, 24000 \\ Campus do Gragoatá, Niterói, RJ, Brasil
}

(Received 25 September 1995 and in revised form 13 May 1996)

An electromagnetic particle-in-cell simulation code is used to investigate particle density effects on the nonlinear amplification of inverse-bremsstrahlung electron acceleration, according to the schemes proposed by Kawata et al. and by Hussein and Pato. It is shown that although the space-charge self-consistent field does not modify the basic results obtained with the one-particle model, the electromagnetic fields produced by the transverse particle current play a substantial role for large beam densities.

\section{Introduction}

Various new ideas for particle accelerators with or without a medium have been proposed in order to overcome the limitation of the acceleration gradient for the current linear accelerators. Kawata et al. (1991) have proposed a scheme for electron acceleration using a laser and a weak perpendicular static electric or magnetic field, the inverse-bremsstrahlung electron-acceleration mechanism. The static applied field breaks the symmetry of the electromagnetic (EM) wave in space and time, allowing the electrons to gain energy in both half-wavelengths of the wave. Hussein and Pato (1992) have analytically solved the coupled electron-EM-field system, which is governed by a set of equations containing one nonlinear and several linear ones. They have shown that the energy gain can be made much larger than that of the Kawata $e t$ al. scheme by employing a system of externally applied electrostatic fields $E_{\text {app }}$, with alternating signs at optimally determined positions. This scheme was dubbed NAIBEA, for "nonlinear amplification of inverse bremsstrahlung acceleration'. The optimal positions were determined by analyzing the expected behaviour of the energy as a function of the phase $\varphi=k(c t-x)$. It was found that the applied field had to be reversed at $\varphi=\frac{1}{2}(2 n+1) \pi$, with $n=1,2,3, \ldots$, in order to get continuous acceleration (Hussein and Pato 1992, 1993). A crucial issue in this scheme is whether the self-consistent space-charge field due to particle bunching affects the critical phase relation between the laser and external fields with respect 
to the particles being accelerated, decreasing the net energy gain. This problem was addressed in a previous work (Galvão et al. 1994) using the so-called phaseaverage bunching approximation for the space-charge self-field (Seo 1991; Serbeto and Alves 1993) and the relativistic beam approximation in the equation of motion of a small number of particles initially uniformly distributed in one wavelength of the electromagnetic field. It was found that the space-charge self-field did not spoil the net energy gain. In this paper we investigate the effect of several other relevant physical processes that have not been properly considered in previous works. Both the schemes of Kawata et al. (1991) and of Hussein and Pato (1992) are simulated using a $1-2 / 2\left(x, v_{x}, v_{y}, v_{z}\right)$ dimensional electromagnetic particle-in-cell simulation code. $\dagger$ Effects such as thermal spread, large beam density, longitudinal electric field, and the electric and magnetic fields generated by the transverse beam current are self-consistently taken into account.

\section{One-particle model}

We briefly present the mechanisms of the inverse-synchroton-radiation acceleration proposed previously (Kawata et al. 1991; Hussein and Pato 1992). Consider an EM wave that propagates in the $+x$ direction at the speed of light $c$. The electric component $E_{y}^{E M}$ is in the $y$ direction and the magnetic component $B_{z}^{E M}$ is in the $z$ direction. Electrons move mainly in the $x$ direction, and their speed is less than $c$. Therefore the EM wave catches up with the electrons and leaves them behind. In this scenario the electrons oscillate and cannot absorb the wave energy. The cause of this oscillation is the symmetry of the EM wave in space and time. Let us now introduce an externally applied static electric $E_{\text {app }}$ (or magnetic $B_{\text {app }}$ ) field. This applied field removes the symmetry of the EM wave in space. The coupled particle-field equations are

$$
\begin{gathered}
\frac{d p_{x}}{d t}=-\frac{e}{c}(\mathbf{v} \times \mathbf{B})_{x}=-e \beta_{y} B_{z}, \\
\frac{d p_{y}}{d t}=-e E_{y}-\frac{e}{c}(\mathbf{v} \times \mathbf{B})_{y}=-e E_{y}+e \beta_{x} B_{z},
\end{gathered}
$$

where $E_{y}=E_{y}^{E M}+E_{\text {app }}$ and $\beta_{i}=v_{i} / c(i=x, y)$, and $p_{x}$ and $p_{y}$ are the $x$ and $y$ components of the linear momentum of the particle. If we now choose $B_{z}^{E M}=$ $E_{y}^{E M}=-E_{y 0} \sin \varphi$, with $\varphi=k(c t-x)$, we have

$$
\begin{gathered}
\frac{d p_{x}}{d t}=-e \beta_{y} E_{y 0} \sin \varphi \\
\frac{d p_{y}}{d t}=e\left(1-\beta_{x}\right) E_{y 0} \sin \varphi-e E_{\mathrm{app}} .
\end{gathered}
$$

The energy equation is

$$
\frac{d \epsilon}{d t}=-e E_{y} v_{y}
$$

It is clear that the speed $v_{y}$ parallel to $E_{y}$ is important for the electron energy gain, and $v_{y}$ is determined by the equation of motion. In order to accelerate an

$\dagger$ The authors are indebted to Dr B. Lembege (Centre de Recherches en Physique de l'Environnement Terrestre et Planétaire, Issy-les-Molineuax, France) for providing a basic version of the particle code used here. 

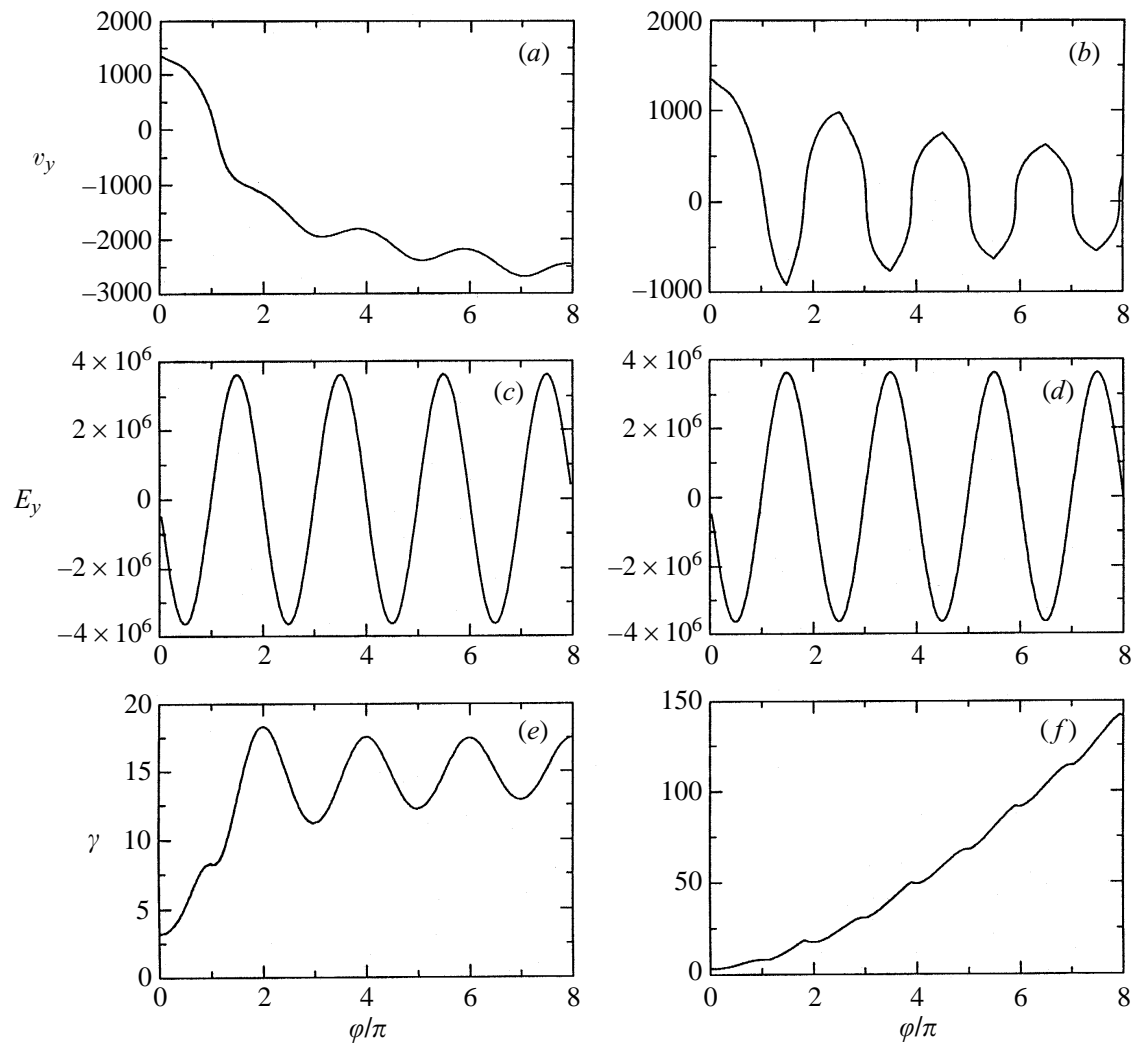

Figure 1. $E_{y}, v_{y}$ and $\gamma$ versus $\varphi$ for the Kawata et al. scheme $(a, c, e)$ and for the Hussein and Pato scheme $(b, d, f)$, from one-particle model. Initial conditions are an electron speed of $0.95 \mathrm{c}\left(\gamma_{0}=3.2\right)$, an incident angle with respect to the $x$ axis of $13.83^{\circ}$ and $E_{\text {app }} / E_{y 0}=2.23 \times 10^{-2}$.

electron in both half-wavelengths in one period of the laser wave, we choose an appropriate value of $E_{\text {app }}$ so that $v_{y}$ becomes zero at $\varphi \approx \pi$; this means that in the half-wavelength $0<\varphi<\pi$ we have $v_{y}>0$, and in the remaining half-wavelength we have $v_{y}<0$. As a result, in the half-wavelength $0<\varphi<\pi$ the electron is accelerated in the $+y$ direction and can absorb wave energy. Then $v_{y}$ decreases according to the force $F_{y}$, and becomes zero at $\varphi \approx \pi$. In the remaining halfwavelength the electron has velocity $v_{y}<0$ and can again absorb wave energy. In addition, the electron is accelerated in the $+x$ direction by the force $F_{x}>0$ in both half-wavelengths, and the interaction between the electron and the EM wave goes on for longer (Kawata et al. 1991). Assume now that we can alternate the sign of $E_{\text {app }}$ at optimally determined locations relative to the phase of the electromagnetic wave. Because of the relativistic effect, the distance between the positions of sign reversal are macroscopic in the laboratory frame. This allows for a continuous acceleration of the electrons (Hussein and Pato 1992). These ideas are illustrated in Figs $1(a-f)$, which show $v_{y}, E_{y}$ and $\gamma$ versus $\varphi$ for the Kawata et al. scheme $(a, c, e)$ and the Hussein and Pato scheme $(b, d, f)$, obtained from the numerical integration of (1) and (2), with the following initial conditions: electron 
speed of $0.95 c\left(\gamma_{0}=3.2\right)$, an incident angle with respect to the $x$ axis of $13.83^{\circ}$, and $E_{\text {app }} / E_{y 0}=2.23 \times 10^{-2}$.

\section{Simulation model}

In the simulation model, particle and field equations are solved in a self-consistent way. To study the mechanisms of electron acceleration, an infinitely long train of electromagnetic radiation with wavenumber $k$ is imposed on an initially uniform thermal electron beam immersed in an static electric field in the $y$ direction. The direction of laser propagation, as well as the allowed spatial variation, is taken to be the $x$ direction. The dynamics of the acceleration process of the particles is described in $(x, \gamma)$ space when we want to observe what is happening to the whole set of particles, or on $(\varphi, \gamma$ (or other variable)) plots when we want to trace the motion of an specific particle.

Let us briefly describe the numerical model of the particle dynamics employed in the simulation code. The trajectories of a large number of particles are followed in time according to the relativistic equation of motion

$$
\begin{gathered}
\frac{d \mathbf{p}_{i}}{d t}=q_{i}\left(\mathbf{E}\left(\mathbf{r}_{i}\right)+\frac{\mathbf{p}_{i} \times \mathbf{B}\left(\mathbf{r}_{i}\right)}{m c \gamma_{i}}\right), \\
\frac{d \mathbf{r}_{i}}{d t}=\mathbf{v}_{i},
\end{gathered}
$$

where $\mathbf{E}$ and $\mathbf{B}$ are the self-consistent plus externally applied electromagnetic fields at the position $\mathbf{r}_{i}$ of the $i$ th particle, and $\gamma_{i}=\left[1+\left(p_{i} / m_{i} c\right)^{2}\right]^{1 / 2}$ is the relativistic factor. From the particle positions and velocities, one calculates the respective charge and current densities on a spatial grid; the corresponding electromagnetic fields are given by the Maxwell equations, namely

$$
\begin{gathered}
\frac{\partial \mathbf{E}}{\partial t}=c \nabla \times \mathbf{B}-\mathbf{J}, \\
\frac{\partial \mathbf{B}}{\partial t}=-c \nabla \times \mathbf{E}, \\
\nabla \cdot \mathbf{B}=0, \\
\nabla \cdot \mathbf{E}=\frac{\rho}{\epsilon_{0}} .
\end{gathered}
$$

Among the different techniques available for solving these coupled equations, we choose the leapfrog method (Birdsall and Langdon 1985; Tajima 1989). In this method (6) is replaced by the finite-difference equations

$$
\begin{aligned}
\frac{\mathbf{p}^{(n+1 / 2) \Delta t}-\mathbf{p}^{(n-1 / 2) \Delta t}}{\Delta t} & =q\left(\mathbf{E}^{n \Delta t}+\frac{\mathbf{p}^{(n+1 / 2) \Delta t}+\mathbf{p}^{(n-1 / 2) \Delta t}}{2} \times \frac{\mathbf{B}^{n \Delta t}}{c \gamma^{n \Delta t}}\right), \\
& \frac{\mathbf{r}^{(n+1) \Delta t}-\mathbf{r}^{n \Delta t}}{\Delta t}=\mathbf{v}^{(n+1 / 2) \Delta t} .
\end{aligned}
$$

In order to solve (12) and (13) we define the auxiliary variables $\mathbf{p}^{+}$and $\mathbf{p}^{-}$by (Boris 1970)

$$
\mathbf{p}^{(n-1 / 2) \Delta t}=\mathbf{p}^{-}-\frac{1}{2} q \mathbf{E}^{n \Delta t} \Delta t
$$




$$
\mathbf{p}^{(n+1 / 2) \Delta t}=\mathbf{p}^{+}+\frac{1}{2} q \mathbf{E}^{n \Delta t} \Delta t .
$$

Introducing these equations into (12), we obtain

$$
\frac{\mathbf{p}^{+}-\mathbf{p}^{-}}{\Delta t}=\frac{q}{2 m \gamma}\left(\mathbf{p}^{+}+\mathbf{p}^{-}\right) \times \mathbf{B}
$$

where $\gamma=\left[1+\left(p^{-} / m_{e} c\right)^{2}\right]^{1 / 2}$, which represents a rotation (assuming that the rotation angle between these two auxiliary variables is small). Hence the computational steps are:

(i) add half the electric impulse to $\mathbf{p}^{(n-1 / 2) \Delta t}$ to obtain $\mathbf{p}^{-}$, which is rotated according to (16) to obtain $\mathbf{p}^{+}$;

(ii) add the remaining half of the electric impulse to obtain $\mathbf{p}^{(n+1 / 2) \Delta t}$.

In solving the Maxwell equations, we have to take into account the applied constant electric field $E_{\text {app }}$, and solve for the self-consistent electromagnetic fields. Since the beam spread in the $y$ direction is not considered, it suffices to calculate only the $x$ component of the self-consistent electrostatic field, $E_{x}=-\partial \Phi / \partial x$. The scalar potential $\Phi$ is obtained by solving the Poisson equation numerically using fast-Fourier-transform techniques assuming periodic boundary conditions. The transverse electric and magnetic fields are obtained from (8) and (9). These equations are solved by decomposing the electric and magnetic fields in left and right-going field quantities that move with propagation velocity $\pm c$. This approach requires that $\Delta x / \Delta t=c$ (where $\Delta x$ is the length of the cell and $\Delta t$ is the time interval for advancing in time), since the equations are integrated along the vaccuum characteristic $x \pm c t=$ constant, and consequently there is no Courant condition in the usual sense (Cohen et al. 1975). The numerical stability depends only on $\Delta t$ and is given by the condition $\omega_{p} \Delta t \ll 1$. The particle and current densities associated with each grid point are calculated from the position and velocity of the electrons using the subtracted dipole scheme (SUD or SUDs) (Tajima 1989).

As initial conditions for the electromagnetic wave we choose $E_{y}^{E M}=B_{z}=$ $E_{y_{0}} \cos k x$ and the particles of the beam are loaded uniformly distributed in position along one laser wavelength. We initiate the velocity of particles in such a way that they have the same spread in $x$ and $y$, determined from the spreads of momenta of the particles, which are obtained from the relativistic Maxwellian distribution (Birdsall and Langdon 1985):

$$
f\left(p^{\prime}\right)=\frac{N m c^{2}}{2 \pi K T} \frac{1}{\left(K T / m c^{2}+1\right)} \exp \left(-\frac{m c^{2}\left(\gamma^{\prime}-1\right)}{K T}\right),
$$

where $N$ is the total number of particles being loaded and $\mathbf{v}^{\prime}=\mathbf{p}^{\prime} / m \gamma^{\prime}$. The function $f$ is normalized by requiring that $\int_{0}^{2 \pi} d \theta \int_{0}^{\infty} f p^{\prime} d p^{\prime}=N$.

To allow a relativistic drift velocity and yet maintain particle velocities less than $c$, the drift velocity $\mathbf{v}_{0}=v_{0 x} \mathbf{i}+v_{0 y} \mathbf{j}$ is added relativistically to each particle velocity $\mathbf{v}^{\prime}$, determined from (17), by

$$
\mathbf{v}=\frac{\mathbf{u}^{+}+\mathbf{v}_{0}}{1+\mathbf{v}^{\prime} \cdot \mathbf{v}_{0} / c^{2}}
$$

where $\mathbf{u}^{+}=\mathbf{v}^{\prime} / \gamma-(1-\gamma) \mathbf{v}_{0}\left(\mathbf{v}_{0} \cdot \mathbf{v}^{\prime}\right) / \gamma v^{\prime 2}$, and $\mathbf{v}$ is the total velocity of each particle of the beam in the laboratory frame. The ions are kept fixed as a uniformly distributed neutralizing background. 

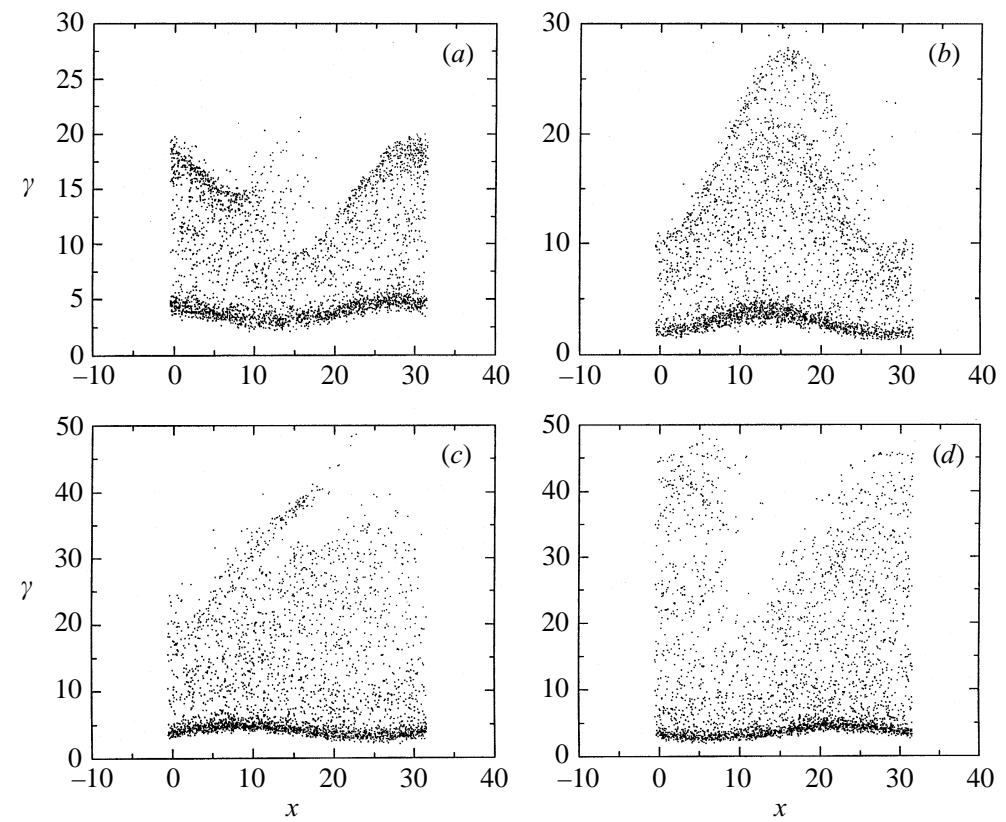

Figure 2. Phase space $(\gamma$ versus $x)$ for the Kawata et al. scheme with $n_{e}=7.96 \times 10^{12} \mathrm{~cm}^{-3}$ and $T=100 \mathrm{keV}:(a) \varphi=2 \pi ;(b) 4 \pi ;(c) 6 \pi ;(d) 8 \pi$. Initial conditions for velocities, positions and electromagnetic fields are described in the text.

\section{Results}

Using the simulation model previously described, we investigate the effect of space charge, beam density and thermal spread. The choice of initial conditions is very critical for efficient particle acceleration, even in the one-particle model. Therefore, to clarify the effects that we are considering, we choose the one-particle optimized conditions already determined by Kawata et al. (1991). The averaged electron speed is $0.95 \mathrm{c}$, the averaged incident angle with respect to the $x$ axis is $13.83^{\circ}$, the amplitude $E_{y 0}$ of the EM wave, $E_{y}^{E M}=B_{z}=E_{y_{0}} \cos k x$, is $1.636 \times 10^{6} / \lambda \mathrm{V} \mathrm{cm}^{-1}$, and applied static electric field $E_{\text {app }}=2.23 \times 10^{-2} E_{y 0}$.

The electrons of the beam are initially considered uniformly distributed along one laser wavelength with a temperature $T$. This distribution is justified, since it is rather difficult to generate an electron beam bunched at the scale of an optical period. Therefore all possible initial phases are equally populated by electrons. We select one particle as a reference particle to obtain $\varphi$. At $t=0$, the position of this particle is $x_{r}(0)=0$ and the value of $\gamma$ is $\gamma_{r 0}=3.2$. For a given time $t, \varphi(t)=k\left[c t-x_{r}(t)\right]$, which is the phase difference between the electric field vector and the motion of the electron along $x$. We remark that the particle distribution is truly relativistic, so that the decay for large energies is less pronounced than for a non-relativistic Maxwellian. We have used $\Delta x=\frac{1}{32} \lambda$ and a total number of simulation particles of 3200 (100 particles per $\Delta x$ ). The initial electron number density is $7.96 \times 10^{6} \lambda^{2} \mathrm{~cm}^{-3}$, which gives $n_{e}=7.96 \times 10^{12} \mathrm{~cm}^{-3}$ for $\lambda=10 \mu \mathrm{m}$, and $T=100 \mathrm{keV}$.

Let us first investigate the effect of high density on the scheme of Kawata et al. The phase space $(\gamma$ against $x$ ) results for different phases of the reference particle, i.e. 

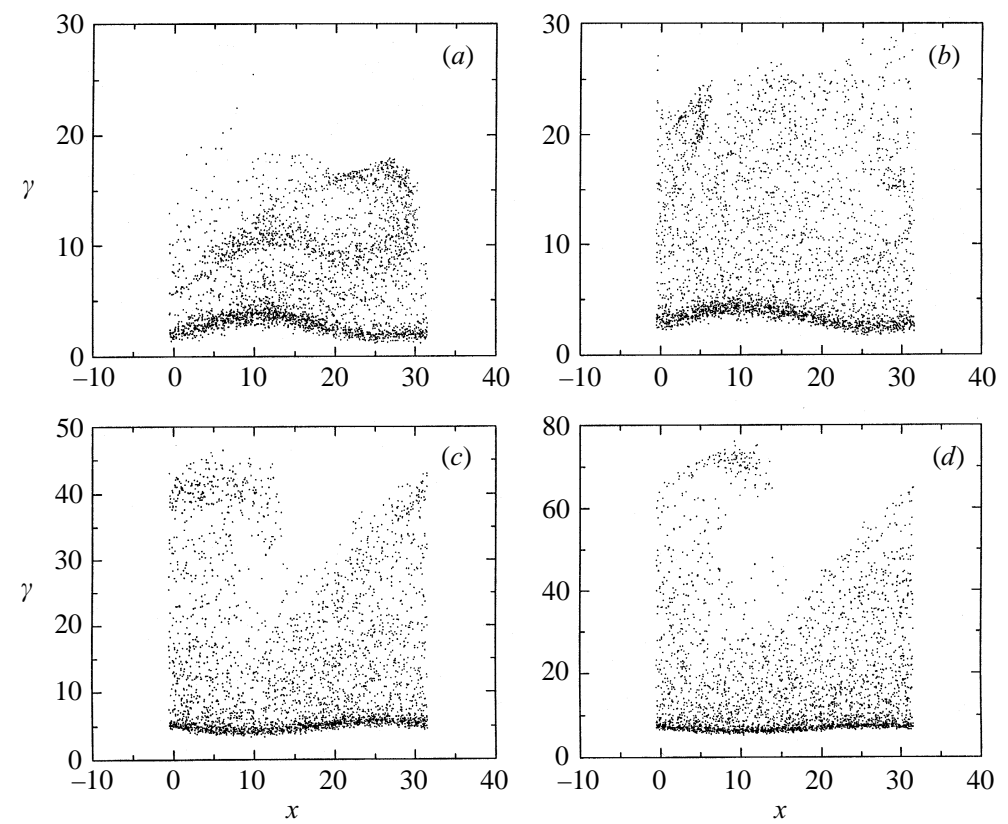

Figure 3. Phase space $\left(\gamma\right.$ versus $x$ ) for the Hussein and Pato scheme with $n_{e}=7.96 \times 10^{12} \mathrm{~cm}^{-3}$ and $T=100 \mathrm{keV}:(a) \varphi=2 \pi ;(b) 4 \pi ;(c) 6 \pi ;(d) 8 \pi$. Initial conditions for velocities, positions and electromagnetic fields are described in the text.

$\varphi=2 \pi, 4 \pi, 6 \pi$ and $8 \pi$, are shown in Figs $2(a-d)$ respectively. The result presented in Fig. 2(a) is to be compared with that shown in Fig. 5 of Kawata et al. (1991). The maximum values of $\gamma$ are of the same order and $20 \%$ of particles have $\gamma>15.0$.

To check the effect of high beam density on the scheme of Hussein and Pato, let us compare the results presented in Figs $3(a-d)$ with the one-particle results (Fig. 1). The phase-diagram plots presented in Fig. 3 are for the same phases of the reference particle as in Fig. 2. Therefore, after seven inversions of the applied field, the phase is $8 \pi$, corresponding to the end of the diagram shown in Fig. $1(f)$. We see that the maximum of $\gamma$ achieved in the one-particle simulation is approximately $\gamma=140$, whereas the maximum value for a beam density $n_{e}=7.96 \times 10^{12} \mathrm{~cm}^{-3}$ is less than $\gamma=80$ (Fig. $3 d$ ). As we discuss below, this effect is not due to the selfconsistent electrostatic field but rather to the electromagnetic field generated by the transverse current $J_{y}$.

In order to understand the reason for these results, not predictable by the oneparticle model, let us consider a smaller beam density, namely $n_{e}=10^{8} \mathrm{~cm}^{-3}$, keeping the same initial values for the other parameters. The results are presented in Figs $4(a, b)$, for $\varphi=8 \pi$ for the Kawata et al. and the Hussein and Pato schemes respectively. We observe that with this low density, the maximum values of $\gamma$ are those predicted by the one-particle model for the Hussein and Pato scheme (Fig. $4 b$ ), and are somewhat larger for the Kawata et al. scheme (Fig. 4a), because some particles at optimized initial positions have $\gamma>3.2$, owing to the thermal spread.

As pointed out before, the sign of the product $v_{y} E_{y}$ is crucial for the process of energy gain. To show that this sign is mainly affected by the field generated by the transverse current $J_{y}$, we have carried out the many-particle simulation, artificially turning off the fields $B_{z j}$ and $E_{y j}$ generated by $J_{y}$. The results are shown in Fig. 5 . 

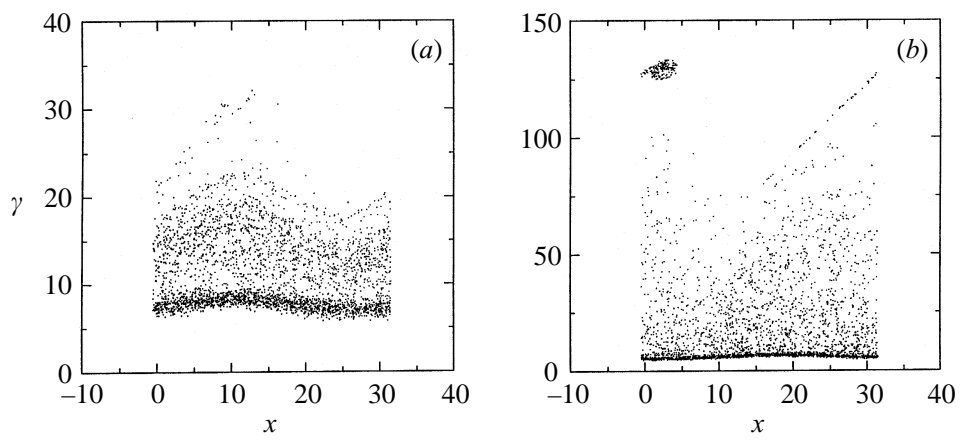

Figure 4. Phase space $(\gamma$ versus $x)$ for $\varphi=8 \pi$, with $n_{e}=10^{8} \mathrm{~cm}^{-3}$ and $T=100 \mathrm{keV}$ : (a) Kawata et al. scheme; (b) Hussein and Pato scheme. Initial conditions for velocities, positions and electromagnetic fields are described in the text.
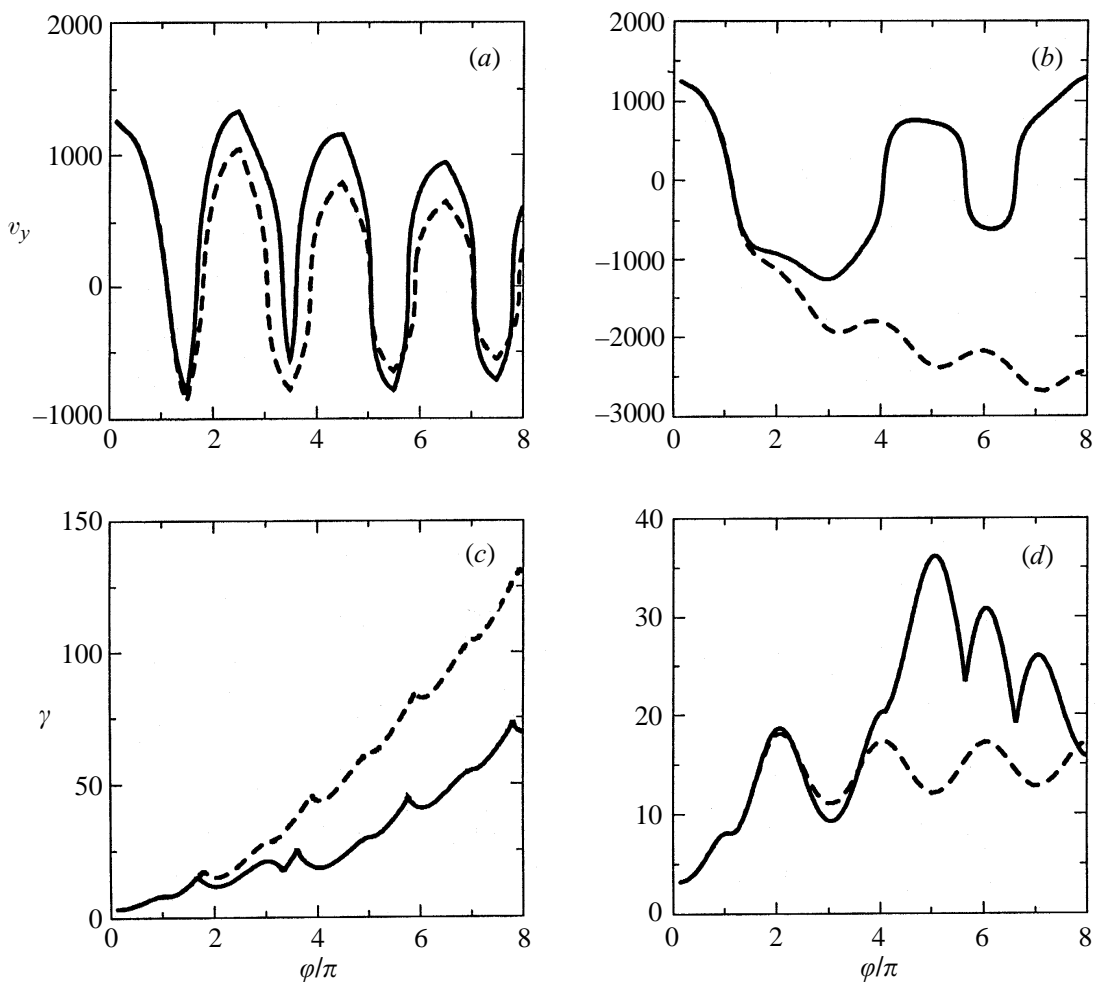

Figure 5. Plots of $v_{y}$ and $\gamma$ versus $\varphi$ for a reference particle in a beam of density $n=7.96 \times 10^{12} \mathrm{~cm}^{-3}:(a, c)$ Hussein and Pato scheme; $(b, d)$ Kawata et al. scheme. Full lines are obtained from a complete many-particle model; dashed lines are obtained when the fields $B_{z j}$ and $E_{y j}$ generated by $J_{y}$ are artificially turned off.

The dashed line corresponds to the fields turned off and the full line to the complete simulation. One can easily verify that results obtained by the one-particle model (Fig. 1) are exactly reproduced by those without the self-consistent electromagnetic fields. We have also verified that the effect of the space-charge electrostatic field is negligible in comparison with the electromagnetic field. 

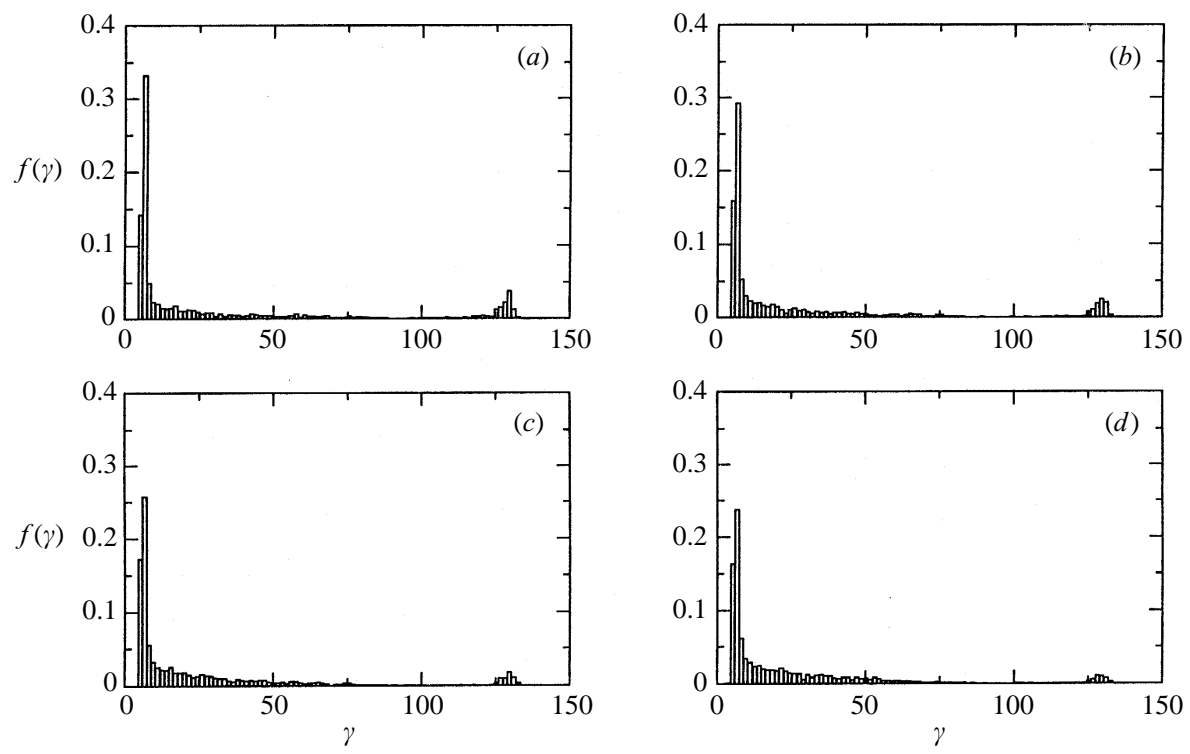

Figure 6. Energy distribution plotted in terms of $\gamma$ as a normalized histogram for different temperatures: $(a) T=10 ;(b) 50 ;(c) 100 ;(d) 150$. Initial density is $n_{e}=10^{8} \mathrm{~cm}^{-3}$ in all cases.

The evolutions of $v_{y}$ and $\gamma$ for the reference particle are shown in Figs $5(a-c)$ respectively for the Hussein and Pato scheme, and in Figs $5(b, d)$ for the Kawata et $a l$. scheme. The evolution of $v_{y}$ is the same as presented in Figs $1(c, d)$, even for the complete many-particle model. Let us first analyse Fig. 5(a) for the Hussein model. We note that $v_{y}$ changes sign together with the applied field $E_{y}$ in the one-particle case, whereas there is a slight phase slippage in the many-particle case. This effect repeats at every change of sign of the applied field, and consequently the overall increase in the value of $\gamma$ is smaller than predicted by the one-particle model, as we can see in Fig. 5(c).

For the Kawata et al. scheme, there is only one change in the sign of $v_{y}$ in the oneparticle model, as shown by the dashed line in Fig. 5(b). We observe that $v_{y}$ changes sign in the complete many-particle model more than once, as indicated by the full line in the same figure. This occurs because the self-consistent electromagnetic field introduces a sign change in the effective fields seen by the particles. As a consequence, the particles gain energy for $\varphi>2 \pi$, as we can see in Fig. $5(d)$. Note however, that this energy gain saturates, and eventually decreases to the one-particle values. Therefore, to tap this extra energy, the acceleration scheme has to be stopped at a phase around $5 \pi$. This mechanism was not identified by Kawata et al. (1991).

We have investigated the effect of the thermal spread of the beam only for the Hussein and Pato scheme, since it presents the best results for the particle acceleration. We consider four different cases, $T=10,50,100$ and $150 \mathrm{keV}$, for high and low density, keeping the same initial values for the other parameters. The results for the low- and high-density cases are shown in Figs 6 and 7 respectively, obtained for $\varphi=8 \pi$. The energy distribution is plotted in terms of $\gamma$ as a normalized histogram. Parts $(a)-(d)$ in both cases are for temperatures $10,50,100$ and $150 \mathrm{keV}$. It is possible to observe beam heating for both the low- and high-density cases. In the low-density case, we also observe a sort of energy bunching for particles with 

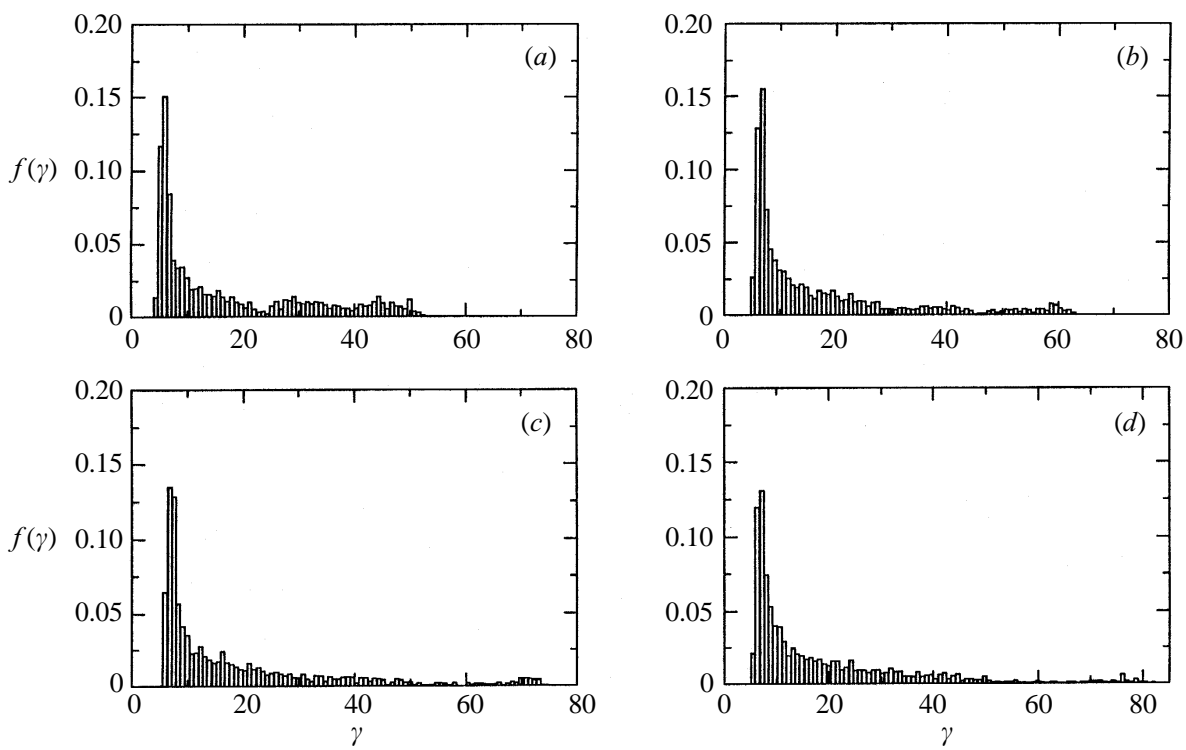

Figure 7. Energy distribution plotted in terms of $\gamma$ as a normalized histogram for different temperatures: $(a) T=10 ;(b) 50 ;(c) 100 ;(d) 150$. Initial density is $n_{e}=7.96 \times 10^{12} \mathrm{~cm}^{-3}$ in all cases.

$\gamma \approx 125$. The number of accelerated particles decreases as the initial beam temperature increases. The thermal spread is higher for the high-density case, and there is no formation of a high-energy beam.

In the low-density case the fields acting upon the particles are basically the laser field and the external field $E_{y}$. The effect of the thermal spread is then only to spoil the optimized initial conditions for the particles in the beam, decreasing the fraction of particles that get accelerated. The thermal effect is less relevant for the high-density case, because of the effect of the self-consistent electromagnetic field, already discussed.

\section{Conclusions}

In this work we have investigated the effect of space charge, beam density and thermal spread on the nonlinear amplification of inverse-bremsstrahlung electron acceleration using particle-in-cell simulation. We observe that the space-charge field due to bunching of the beam plays a negligible role. Varying the beam thermal spread can change the fraction of accelerated particles in the low-density case. We find that the most important effect is due to the electromagnetic fields generated by the beam that appear when the density is sufficiently high. These fields significantly alter the behaviour of the tranverse particle velocity, modifying the results predicted by the one-particle model in the Hussein and Pato (1992) and Kawata et al. (1991) schemes. In the high-beam-density case the effect of the self-consistent electromagnetic field dominates the thermal-spread effect.

\section{Acknowledgements}

This work was partially supported by the following Brazilian Agencies: CNPq (Conselho Nacional de Desenvolvimento Cientifico e Tecnologico), FAPESP (Fundação 
de Amparo à Pesquisa do Estado de São Paulo) and FINEP (Financiadora de Estudos e Projetos). We are grateful for stimulating conversations with Professor G. Morales. One of the authors (R.M.O.G.) acknowledges Dr P. Sprangle for fruitful discussions.

\section{References}

Birdsall, C. K. and Langdon, A. B. 1985 Plasma Physics via Computer Simulation. McGrawHill, New York.

Boris, J. P. 1970 Relativistic plasma simulation-optimization of a hybrid code. Proceedings of 4th Conference on Numerical Simulation of Plasmas, Naval Research Laboratory, Washington, DC, 2-3 November $19 \% 0$.

Cohen, B. I., Monstrom, M. A., Nicholson, D. R., Kaufman, A. N., Max, A. E. and Langdon, A. B. 1975 Phys. Fluids, 18, 470 .

Galvão, R. M. O., Hussein, M. S., Pato, M. P. and Serbeto, A. 1994 Phys. Rev. E49, R4807.

Hussein, M. S. and Pato, M. P. 1992 Phys. Rev. Lett. 68, 1136.

Hussein, M. S. and Pato, M. P. 1993 Int. J. Mod. Phys A8, 3235.

Hussein, M. S., Pato, M. P. and Kerman, A. K. 1992 Phys. Rev. A46, 3562.

Kawata, S., Maruyama, T., Watanabe, H. and Takahashi, I. 1991 Phys. Rev. Lett. 66, 2072.

Seo, Y. 1991 Phys. Fluids B3, 797.

Serbeto, A. and Alves, M. V. 1993 IEEE Trans. Plasma Sci. 21, 243.

Tajima, T. 1989 Computational Plasma Physics with Applications to Fusion and Astrophysics. Addison-Wesley, Reading, MA. 